\title{
Associations of interleukin-17 and monocyte chemoattractant protein-1 with vascular lesions in patients with rheumatoid arthritis.
}

\author{
Zhaocui Zhang $^{1 *}$, Shuhong Zhou ${ }^{1}$, Yi Zhang ${ }^{2}$ \\ ${ }^{1}$ Department of Rheumatism, Gansu Provincial People's Hospital, Lanzhou 730000, PR China \\ ${ }^{2}$ Department of Neurology, Gansu Provincial People's Hospital, Lanzhou 730000, PR China
}

\begin{abstract}
This study aimed to investigate the associations of Interleukin-17 (IL-17) and monocyte chemoattractant protein-1 (MCP-1) with vascular lesions in patients with rheumatoid arthritis (RA). Thirty RA patients (RA group) and thirty healthy subjects (control group) were enrolled in this study. In all subjects, the serum levels of rheumatoid and inflammation related indexes including rheumatoid factor (RF), erythrocyte sedimentation rate (ESR), tumor necrosis factor- $\alpha$ (TNF- $\alpha$ ), highsensitivity C-reactive protein (hs-CRP), IL-17 and MCP-1 were determined. In addition. the brachial-ankle pullse wave velocity (baPWV) and ankle-brachial index (ABI) were measured. Rcovlts showed that, the levels of ES, $\mathbb{R F}$, serum TNF- $\alpha$, hs-CRP, IL-17 and MCP-1 in RA group were signticanly higher those in control group, respectively $(\mathbf{P}<0.05)$. The levels of serum TNF- $\alpha$, hs-CRR $\mathbb{1} 17$ and MCP-1 in $\mathbb{R A}$ patients with abnormal baPWV (>1400 cm/s) were significantly higher those in R patients with normal baPWV ( $1400 \mathrm{~cm} / \mathrm{s})$, respectively $(\mathbb{P}<0.05)$. The levels of serum hs-CRA IL- $M$ and MCP-1 in $\mathbf{R A}$ patients with abnormal ABI $(\leq 0.9)$ were significantly higher pose in patients with normal ABI $(>0.9)$, respectively $(\mathbb{P}<0.05)$. In $30 \mathrm{RA}$ patients, hs-CRP, If 17 and $\mathrm{MCP}-1$ were the main independent risk factors of RA patients with abnormal baPWV, and II and MCP-1 were the main independent risk factors of RA patients with abnormal ABI. In conctysion, IL-17 and MCP-1 are involved in the occurrence and development of RA, and theyre be reliable indicators for judging the vascular lesions in $\mathbb{R A}$.
\end{abstract}

Keywords: Rheumatoid arthritis, Vascula lesion Interleukin-17, Monocyte chemoattractant protein-1.

\section{Introduction}

Rheumatoid arthritis (RA) is a systenc autoimmune disease
which is typically characterized by chronic symmetric nonsuppurative arthritis. It seriously affects the health and life quality of patients. The main clinical manifestations of RA include joint involvement, weight loss and fatigue and other symptoms. For severe cases, the function of joint is seriously affected $[1,2]$. Vascular lesion is one of the manifestations of extra-articular lesions of RA, which is mainly characterized by vessel wall invasion of lymphocytes, neutrophils and plasma cells, intimal hyperplasia, fibrin necrosis or thrombosis $[3,4]$. The cardiovascular events account for $42 \%$ of the cause of death in RA patients [5]. The atherosclerosis is the main lesion in the cardiovascular events of RA [6]. Previous studies have shown that, the increase of vascular stiffness is closely related to the mortality rate of cardiovascular events $[7,8]$. It is found that, the arterial stiffness exists in patients with RA [9]. The early assessment of the vascular lesions in RA patients and the related early intervention are important for improving the prognosis of RA. At present it is agreed that the inflammatory response is a common pathway of atherosclerosis occurrence

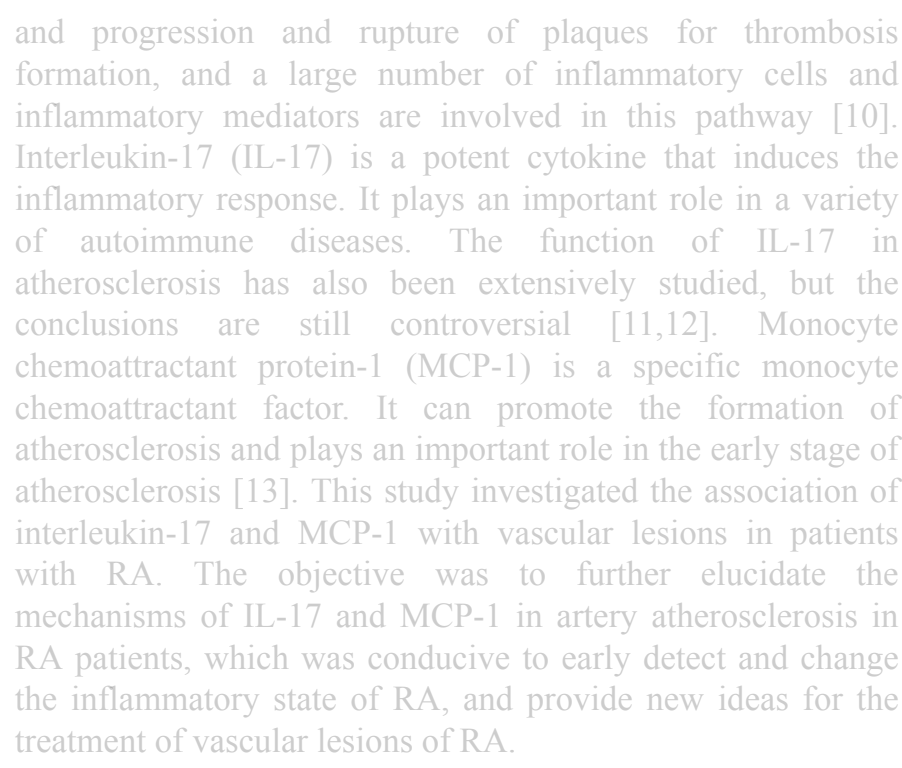

